\title{
Crowd-sourcing Task Research Based on Big Data --Math Model of Pricing Done
}

\author{
Chenghui Yang ${ }^{1,2,3,4}$, Ning Cai ${ }^{1,2}$, Xing Peng ${ }^{5}$, Zhongfu Luo ${ }^{5}$ and Zeyi Liu ${ }^{5}$ \\ ${ }^{1}$ College of Electrical Engineering, Northwest Minzu University, No. 1, Northwest Xincun, Lanzhou, Gansu 730030, China \\ ${ }^{2}$ China National Institute of Information Research, No. 1, Northwest Xincun, Lanzhou, Gansu 730030, China \\ ${ }^{3}$ School of Automation and Electrical Engineering Lanzhou Jiaotong University, No. 188, Lanzhou, Gansu 730070, China \\ ${ }^{4}$ Gansu Artificial Intelligence and Graphic Image Processing Engineering Research Center, Lanzhou, Gansu 730070, China \\ ${ }^{5}$ School of Civil Engineering, Northwest University for Nationalities, No. 1, Northwest Xincun, Lanzhou, Gansu 730030, China
}

\begin{abstract}
Aiming at practical situations, multiple tasks may be because the location is relatively concentrated, leading users will choose, is a consideration of these tasks jointly issued together package. In such case, the pricing model of how to modify the front, to the final completion of the mission and what's the effect? In two under the premise of the model taken together, the task Crowd-sourcing issued to members of the APP program, combined with the Crowd-sourcing mode of operation of the process, the use of reverse guide price method, get a new pricing method, Shenzhen Baoan District City, Shenzhen Luohu District City, Nanhai District of Foshan city in the more populous intensive article using the above Crowd sourcing pricing at In the less expensive areas, the pricing game between members and task providers is adopted.
\end{abstract}

Keywords—crowd sourcing; reverse pricing method

\section{INTRODUCTION}

“Take money" is a kind of self-help service. The user can download the registration become a member of APP, and then receive the task need to take pictures from the APP, complete the task can earn APP calibration to complete the task of remuneration. This self-service service based on mobile Internet Crowd sourcing platform, provide a variety of commercial inspection and information gathering for the enterprise, compared to the traditional market survey method can greatly reduce the cost of the investigation on the one hand, many APP members can easily make the calibration of the remuneration and access to a large number of members, on the other hand, effectively ensure data authenticity and reliability, but also shorten the research cycle.

Therefore, APP has become the core of the platform operation, and the task pricing in APP is its core element. If the price is unreasonable, some tasks will be nobody interested, and lead to the failure of commodity inspection. We need to discuss the pricing rule from China, and propose solutions.

\section{DESIGN IDEAS AND METHODS}

In the premise of the second model, take the task together Crowd sourcing issued to members of the APP program, combined with the Crowd sourcing mode of operation of the process, the use of reverse guide price method, new pricing,
Bao'an District, Shenzhen Luohu District, Nanhai District in more densely populated by the the above Crowd sourcing pricing, the membership and task provider pricing game in the way of task less place. Using the hypothesis of the data of all true, in accordance with the actual local situation. Ignoring the local weather, special effects and other aspects of the psychological data caused by malicious tasks and violation of the platform. The provisions of the act without.

\section{MODEL IMPLEMENTATION}

Model two:

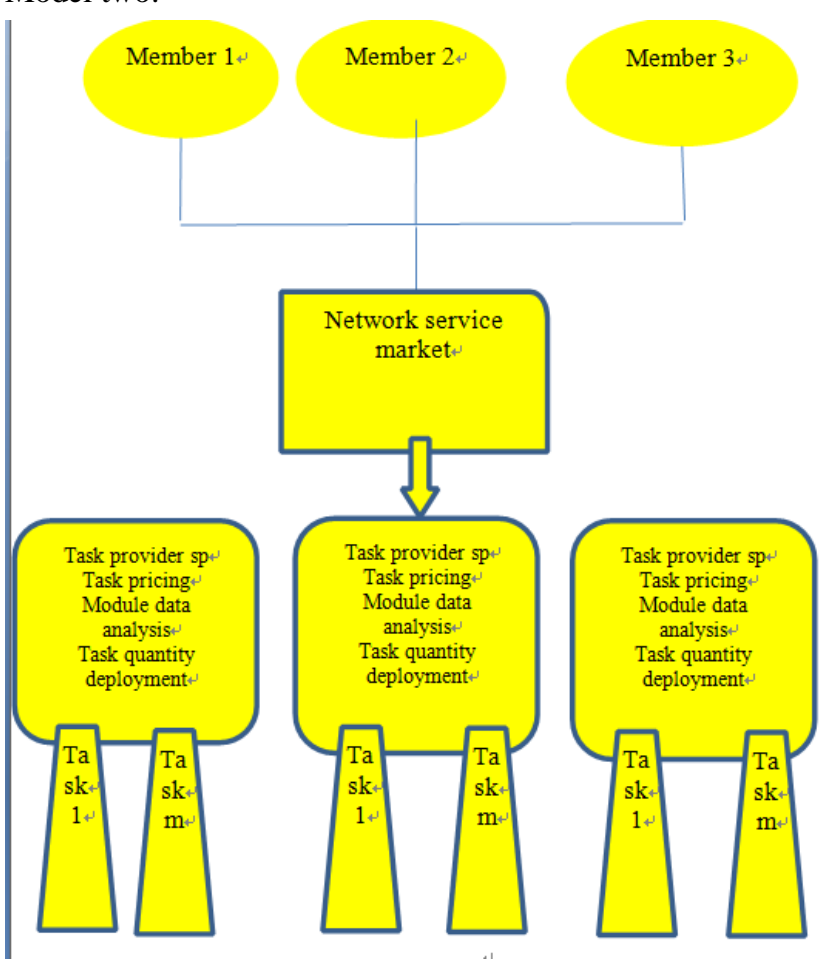

FIGURE I. RELATIONSHIP BETWEEN MEMBER AND TASK

\section{A. The Establishment and Solution of Problem Three}

In model two, there will be a number of tasks may be because the location is relatively concentrated, leading users 
will choose a scheme is adopted these tasks together Crowdsourcing released to the APP member, in the modern enterprise is a mainstream Crowd-sourcing way, the problem of collective packaging and Crowd-sourcing task service mode is the same. Behind the use of Crowd-sourcing to solve and discuss the modifications of the new pricing.

\section{1) Crowd-sourcing explanation}

Crowd-sourcing refers to a company or institution of the past tasks performed by the employee, free and voluntary form of outsourcing to non-specific public network approach. Crowd-sourcing tasks are usually borne by individuals, but if that involves many people work together to complete the task, there may appear in the individual production in the form of relying on open source, method of the open source code is applied to various fields including software [7].

The rise of Crowd-sourcing caused by production changes, "take money" through the realization of APP Crowd-sourcing the traditional geographical interval cannot be achieved efficiently organized a large number of willing to earn rewards APP membership, concentrated to complete the task, to overcome traffic, people are scarce, sold at a low price in the form of combination, put into production in a certain range.

2) The operation process of crowd-sourcing mode

According to whether the target task can be integrated in the operation mode, the source code type and non open source code task can be separated.

a) The open source code [7]: task operation process is composed of a proposed task to build a platform for gathering in a certain region provided to the process required APP membership task professional photos. The task of open source code works with complementary, with the increase of the collection value increases, which integrated all the works as a whole after the increase in the value of individual works. Such as youku.com and tudou.com, gallery. Profit operation process is relatively simple, first of all, this task release task; then, APP members will be uploaded to the photo achievement task put forward to build the platform, as long as it meets the requirements of this task can be obtained Remuneration.

b) The non open source task operation process: tasks proposed release task, process of collecting for the photos and works in a number of APP members plan to choose the most satisfactory work. Non open source task the works between competitive and alternative, the main representative of zhubajie.com, K68.cn and so on. The specific process it can be roughly divided into the bidding process and task process two [8]. membership map.

The non open source task operation process: tasks proposed release task, process of collecting for the photos and works in a number of APP members plan to choose the most satisfactory work. Non open source task the works between competitive and alternative, the main representative of zhubajie.com, K68.cn and so on. The specific process it can be roughly divided into the bidding process and task process two [8]. membership map.
The bidding task flow is generally applied in the relatively complex, high-tech fields. This process has a certain number of screening and works is restricted to participate in membership, work quality has been increased accordingly. First of all, task publishing tasks; then, the task release screening registration member; then the members to submit works; finally, put forward the task release screening and pay the process as shown in Figure 2.
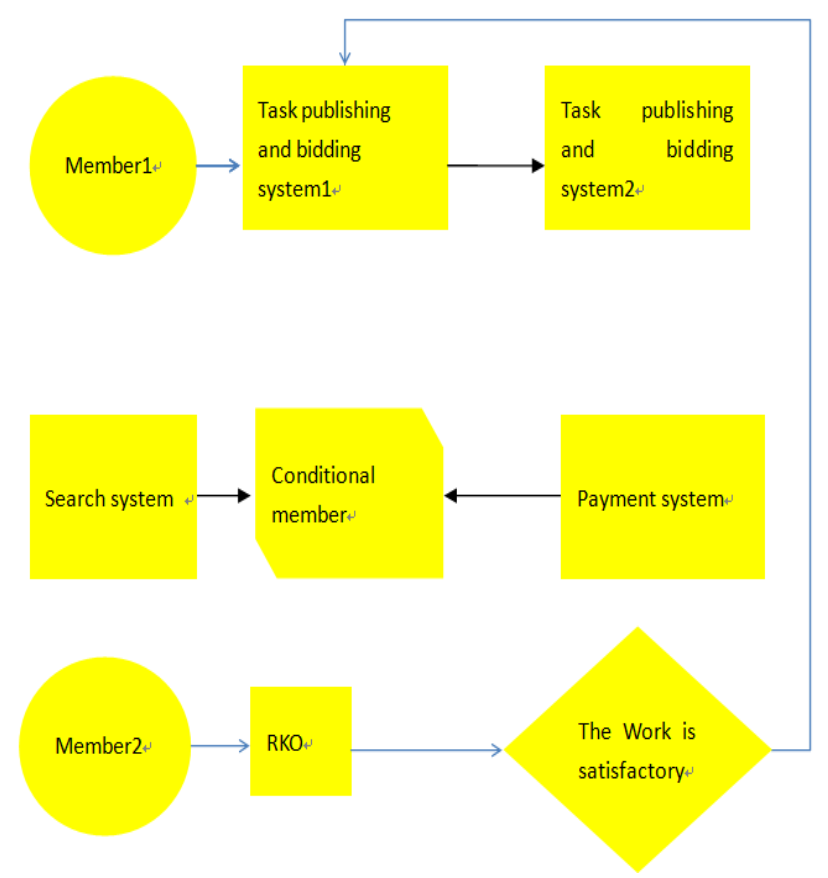

FIGURE II. FLOW CHART OF FINISH THE TASK

Membership map process is applicable to more sophisticated tasks, membership map to member platform opened a personal space for each member of the APP, so as to achieve publicity and the members of their own make money this model. This model can not only reduce the cost of the task publisher and can attract more high-level talents to join the ranks of members, and members of the APP platform through the membership map derivatives such as profitability, trading commissions, advertising or PPC, complete the task of reputation value pricing. Firstly, APP members set up personal space in membership platform; then put forward the task release query by keyword search to AP $\mathrm{P}$ members; then the two sides get in touch and decide whether to cooperate; finally, the transaction is completed and evaluated on the member platform, which further reflects the importance of reputation value. The process is shown in figure 3 . 


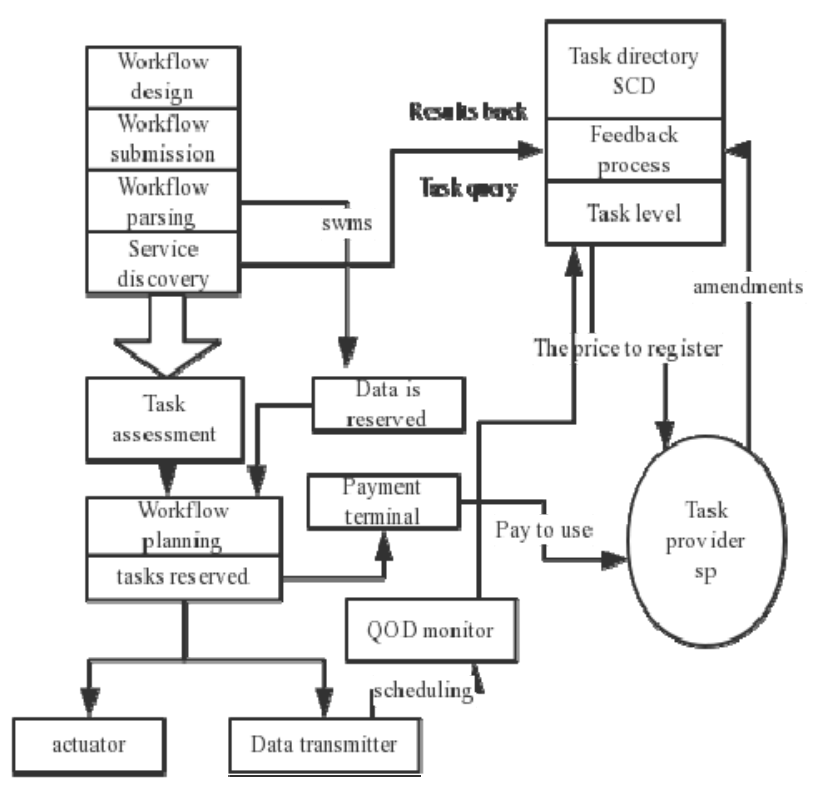

FIGURE III. MEMBER MAP FLOW CHART

\section{3) New pricing method}

More focus on location based on the user will choose Crowd-sourcing released to get the APP members to accept the task, members can Crowd-sourcing way to obtain the inverse oriented price method of remuneration. Use of cost oriented pricing requires accurate accounting of the total cost of the $\mathrm{C}$ task, and then add a certain target profit $\mathrm{Pi}$ the number of photos, the task $\mathrm{Q}$ divided by the service price, the

calculation formula is as follows: $\mathrm{P}=\frac{C+\pi}{Q}$.However, it is difficult to calculate the task cost accurately, especially the personalized task product is difficult to scale. The task price elasticity Ed is also considered. Assuming the pre adjustment price is P0, the pricing formula is as follows: $\mathrm{P}=\frac{1}{2}\left(3-\frac{1}{|E|}\right)$,Suppose he demand function of task outcome is: $P=\alpha+\beta \chi, \alpha, \beta \geq 0$,Pc is the price of other tasks. The beta represents the influence weight of the former price on the demand task, and the theta represents the weight of the recent task price change. The gamma represents the weight of the impact of the flat offer of crowd sourcing members on demand. Therefore, the long-term task price strategy can be concluded as follows:

$$
\lim _{n \rightarrow \infty} P_{n}=\frac{\alpha-\gamma+\theta+C \beta+\frac{C \theta}{P_{C}}+\sqrt{\left(\alpha-\gamma+\theta+C \beta+\frac{C \theta}{P_{C}}\right)^{2}+8 C \gamma\left(\beta+\frac{\theta}{P_{C}}\right)}}{2+\left(\beta+\frac{\theta}{p_{C}}\right)}
$$

The task of product of task value of influence the perceived value of products and reputation to rely more on the cost of the task. The task results of higher quality, full of higher pricing and pricing will work with other members of Crowd sourcing pricing fluctuation changed.

In this way, the average price of the outsourcing industry is positively related to the price of the task publisher, the difficulty of the task, the duration, the platform similarity task of the members, the number of members of the platform have a positive impact on the job publisher's bid.

\section{CONCLUSION}

By using Crowd sourcing model and inverse method to set price oriented remuneration, significantly improved the gathering tasks and task completion efficiency, task providers results in the inspection of members of the examination, the remuneration paid after obtaining approval, and to the members of APP evaluation, obtain reputation value. Later in the mission, the use of high reputation value to accept more tasks, task providers also convenient choice by the who completed the task to improve the completion rate and save a lot of time. To every single task can be completed and members, will be successful. In terms of remuneration between the members and the task of the provider, the two sides is to maximize the benefits, in the difficult task, the number and the pricing, the two sides can negotiate a member can compete with each other. In this mode, Shenzhen City, Baoan District Shenzhen, Luohu District City, Nanhai District, Foshan City, the unfinished task of the amount will be less and less, the population more densely with more than Crowd-sourcing the pricing, the price will be lower, the membership and task provider pricing game way in the task of the place, the same members can also get rich rewards.

\section{ACKNOWLEDGEMENT}

[1] 2017 “13th five-year plan” education scientific planning key project of gansu province "advanced education targeted poverty alleviation research"(Item no.: GS [2017] GHBZ034);

[2] Northwest national university 2017 central university project fund.(Item no.: 31920170079);

[3] Research and practice on the course process reform of digital electronic technology in northwest nationalities university in 2017. (Item no.: 2017XJJG-22);

[4] In 2017, the special fund for basic scientific research operation of the central university of northwest nationalities university. (Item no.: 31920170141);

[5] In 2016, the youth team of the central university project fund of northwest nationalities university. (Item no.: 31920160003);

[6] Supported by Program for Changjiang Scholars and Innovative Research Team in University (IRT_16R36)

[7] In 2018, Northwest University for Nationalities opened the laboratory project, Central University (SYSKF- 
2018002; SYSKF-2018007; SYSKF-2018030; SYSKF2018025)

[8] Supported by Program of 2018 Scientific research project of colleges and universities of Gansu province department of education, "advanced education targeted poverty alleviation research": (Item no.: 2018B-026)

\section{REFERENCES}

[1] Perfect membership marketing model on [J]. field. 2016. Modern commercial Mitsuoki (32).

[2] Wu Zhihua,Shandong Ginza shopping mall VIP customer marketing strategy [D], Xi'an University of Science And Technology, 2015.

[3] Cai Li, "SKYWORTH cool mall membership marketing research [D],”Huazhong University of Science and Technology, 2013.

[4] Yuan Yuan, "Membership marketing model based on customer value [D]," Ocean University of China, 2012.

[5] Wu Wei, "Membership marketing research from the perspective of communication [D]," Soochow University, 2010.

[6] Zhang Hongtao, "Information technology to help retail industry, improve membership marketing [J],”Modern Industry, 2008, (22): 5053. [2017-09-17]. DOI:10.14097/j.cnki.5392/2008.22.013

[7] Yu, RBuyya, “An taxonomy of workflow management systemsForgridcomputing[J],” Journal ofGrid Computing,2005,3(3-4): 171- 200.

[8] Article in a journal:

[9] Yu, R Buyya,C K Tham, "Cost-based scheduling of scientificworkflow applications on utility grids[A].Proceedings of the1st IEEE International Conference on e-Science and Grid Computing[C],” Melbourne Australia:IEEE,2005.140-147.

[10] Jin Qingqing, Chan Weining, Zhou Minchi, Zhou Ao-ying. Benchmark for data management systems: from traditional databases to emerging large data [J]. Journal of Computer Science, 2015,38 (01): 18-34

[11] Shu Wenying. Investigation and analysis on the present situation of impoverished students in Gansu Province [J]. Science and technology Higher Education Research, 2009,28 (02): 77-80

[12] Hu Shuxiang, Sheiyu. Network ideological and political education in large data age [J]. Ideological education Research, 2013 (6)

[13] Dong Yang, Miao Jingsen. Thinking of poverty alleviation in large data age [J]. Cooperative economy and Technology, 2016, (14): 189190

[14] Wu Chaowen, Jin, Sun Yannan. A study on the precision subsidy model of impoverished college students in large Data environment [J] Heilongjiang higher education Research, 2016, (12): 41-44. 